\title{
PARIR EN CASA EN TIEMPOS DE CORONAVIRUS
}

Home Birth in Times of COVID-19

Silvia Costa Abós $^{1}$, Mahault Behaghel ${ }^{2}$

Autora correspondencia: Silvia Costa Abós

Correo electrónico: scosta@ub.edu

1. Doctora en Enfermería. Departamento de Enfermería Fundamental y Medicoquirúgica. Facultad de Medicina y Ciencias de la Salud. Universidad de Barcelona. (Barcelona, España)

2. Matrona autónoma especializada en el seguimiento global y la atención de partos en casa. (Barcelona, España)

Recibido: 03/06/2020

Aceptado: 15/07/2020

(c) (i) 8 


\section{RESUMEN}

A las incógnitas que habitualmente rodean al acto del nacimiento, se añaden ahora nuevas incertidumbres por la pandemia de la COVID-19. Incertidumbre sobre cómo va a afectar a los partos la saturación de los servicios sanitarios o cuál es la mejor manera de actuar. Los protocolos cambian de la noche a la mañana y en algunos hospitales las mujeres gestantes son obligadas a parir en soledad o son separadas de sus bebés nada más nacer. Por todo ello, muchas gestantes a punto de dar a luz están viviendo muy angustiadas la etapa final de sus embarazos. Ante esta situación, no es de extrañar que las peticiones de tener el parto en casa se hayan triplicado en este momento. El objetivo de este trabajo es dar voz a las mujeres, y por ello describimos la experiencia de una mujer gestante que decidió parir en su casa cuando se encontraba casi al final de su embarazo y su vivencia de todo el proceso. A través de una entrevista en profundidad, con varios encuentros, se obtiene un relato profundo y secuencial a partir de la semana 38 de gestación hasta el alumbramiento, que tuvo lugar el 13 de abril de 2020. La experiencia de parir y de acompañar un parto se interpreta como una demostración de las capacidades humanas, de la energía y de las potencialidades de cada persona. Tras esta crisis, consideramos que las autoridades sanitarias deberían replantearse si los hospitales son el mejor escenario para un parto. Una tarea más a la que tendremos que enfrentarnos en un futuro, esperemos, no muy lejano.

Palabras clave: parto en casa; matrona, COVID-19.

\section{ABSTRACT}

To the unknowns that usually surround the act of birth, new uncertainties are now added due to the COVID-19 pandemic. Uncertainty about how the saturation of health services will affect your deliveries or what is the best way to act. The protocols change overnight and in some hospitals they are forced to give birth alone or are separated from their babies as soon as they are born. For all this, many pregnant women about to give birth are experiencing the final stage of their pregnancies very distressed. It is not surprising that requests to give birth at home have tripled at this time in Catalonia. The aim of this work is to describe the experience of a pregnant woman who decided to give birth at home almost at the end of her pregnancy and her experience of the whole process. Through the in-depth interview, carried out at various times, the sequential account is obtained from the 38th week of gestation until delivery, which took place on April 13, 2020. The experience of giving birth and accompanying a birth is interpreted as a demonstration of the human capacities, energy and potential of each person. After this crisis, the health authorities should rethink whether hospitals are the best setting for delivery. One more task that we will have to face in the future, we hope, not too far away.

Keywords: Home Birth; Midwives; COVID-19. 


\section{INTRODUCCIÓN}

En los últimos años, el aumento del uso de la tecnología sanitaria ha mejorado los resultados perinatales, pero se ha mantenido la tendencia a atender de la misma forma todos los partos, tanto los de bajo riesgo como los de alto, lo que ha supuesto más intervencionismo y medicalización, y que los deseos de las mujeres con frecuencia se subordinen a las prioridades organizacionales (FAME, 2011). De hecho, en España, casi uno de cada cuatro nacimientos, un 25 \% del total, se produce mediante cesárea, mientras que la OMS (2015) considera que una cifra justificable no debería superar el $10 \%$ o el 15 \%. El número de partos instrumentales es también elevado, con uno de los porcentajes más altos de Europa (Vivas, 2020).

En la actualidad, existe una cierta controversia acerca del mejor lugar donde parir, ya que se está produciendo un leve retorno al parto domiciliario debido al alto nivel de intrusión en el parto normal, lo cual puede generar insatisfacción en la mujer y en su familia con respecto a la experiencia del parto. Hay un sector de la población que pide una atención al parto más personalizada y participativa, ya que quieren que el nacimiento de sus hijos/hijas suceda en un entorno donde sea posible un parto sin intervenciones, en un ambiente agradable, pero con las garantías de seguridad de un hospital (FAME, 2017). Esta tendencia polemiza la medicalización y la aplicación de altas tecnologías en un proceso biológico, haciendo que el parto se transforme y deje de ser un evento fisiológico (Goberna, 2009; Ramírez, 2015; Henshall, Taylor y Kenyon, 2016; Biurrún, 2017; Araújo et al., 2019).

La atención al parto en el Sistema Nacional de Salud español solo está contemplada en el medio hospitalario. Esto no ocurre como en otros países, que tienen centros de nacimiento dirigidos por matronas fuera de los hospitales, aunque próximos a ellos. Dar a luz en estos centros, además de disminuir el riesgo de un contagio aleatorio, ofrece una experiencia de parto óptima en un ambiente menos medicalizado que un hospital (Ruiz Berdún, 2020). Otra de las posibilidades en algunos países de nuestro entorno es la financiación de la atención al parto en casa para aquellas mujeres que lo deseen. Incluso algunos países, entre ellos Reino Unido y Holanda (KNOV, 2020), cuentan con personal específico para ello. Algo que contrasta con nuestro país, en el que constantemente se nos recuerda la peligrosidad del parto a domicilio, a pesar de que la evidencia científica dice lo contrario, admitiendo la iatrogenia de algunas intervenciones obstétricas y por lo tanto 
la menor producción de riesgos si estas no se efectúan. Algunos ejemplos pueden ilustrar esta afirmación. La demostración de que el rasurado del vello púbico no evita infecciones, que la episiotomía no reduce el riesgo de desgarros, que la posición de litotomía no favorece el expulsivo, la anestesia epidural no beneficia las contracciones uterinas, la administración de oxitocina a la madre no ayuda al bienestar fetal, y así sucesivamente, es lo que ha inducido a promover algunos cambios (Pinto, 2016; Triuzzi, 2017).

La pandemia de coronavirus (Culp, 2020) ha traído un escenario inédito para las embarazadas y parturientas, así como para los recién nacidos, sus padres y familias. Los mensajes de alarma, la preocupación, el miedo a la infección, las recomendaciones cambiantes y la infodemia o exceso de información suponen un estrés añadido muy importante a la hora de dar a luz e iniciar la lactancia (Olza, 2020). A las incógnitas que habitualmente rodean el acto del nacimiento, se añaden ahora nuevas incertidumbres por la crisis del COVID-19 (O’Connell et al., 2020). Incertidumbres sobre cómo va a afectar a sus partos la saturación de los servicios sanitarios o cuál es la mejor manera de actuar. Muchas gestantes a punto de dar a luz han vivido muy angustiadas la etapa final de sus embarazos (Franco, 2020; Cores, 2020).

La presión asistencial y la sobresaturación de los hospitales, así como la falta de experiencia sobre el comportamiento de este virus, han dado lugar en muchos centros a un cambio drástico en los protocolos, que se ha traducido, por una lado, en un mayor intervencionismo obstétrico, con mayores tasas de inducciones, estimulación del parto, partos instrumentales y cesáreas, y por otro, en la limitación o ausencia de acompañante durante el parto o separaciones madre-recién nacidos muy traumáticas y raramente necesarias (Olza, 2020). En varios hospitales de la Comunidad de Madrid, se creó un protocolo de atención al parto según el cual «Dado que durante los pujos activos la exhalación es mayor y no se puede asegurar la efectividad de las mascarillas, se intentará acortar lo más posible la fase activa del expulsivo (kiwi, fórceps...)», una situación que según la abogada Fernández Guillén atenta contra la integridad física de las gestantes y contra los derechos humanos (Fernandez, 2020).

La pandemia ha aumentado el riesgo de sufrir violencia obstétrica y trauma en el parto (Martínez-Mollá, Siles y Solano, 2019). Si en circunstancias normales una de cada tres madres sale del parto con un trauma psíquico, y un $5 \%$ de todas las madres sufren un síndrome de estrés postraumático, esas cifras se han visto incrementadas considerablemente durante la pandemia Covid (Olza, 2020). Podemos citar el testimonio 
de dos mujeres que dieron a luz en pleno confinamiento: Ambas coinciden en su agradecimiento por la asistencia que han recibido: «Absolutamente cuidadosa y atenta», explica Isa. «Un trato excelente; yo creo que me arroparon aún más al ver que, por un malentendido, había ido sola a dar a luz», cuenta Ana. Un parto en unas circunstancias muy especiales, cuyo mayor exponente fue el reencuentro con su marido: «Te emocionas, pero mantienes la distancia. Él entró en la habitación y lo primero que hizo fue echarse líquido desinfectante por todas partes, antes casi ni de mirarnos» (EFE, 2020).

Estas prácticas van en contra de las recomendaciones de la Organización Mundial de la Salud (OMS, 2014). Este organismo especifica que todas la embarazadas, incluso cuando se sospeche o se haya confirmado que tienen la COVID-19, tienen derecho a tener una experiencia de parto positiva, es decir:

1. Ser tratada con respeto y dignidad.

2. Estar acompañada por una persona de su elección durante el parto.

3. Comunicación clara del personal del servicio de maternidad.

4. Estrategias adecuadas de alivio del dolor.

5. Movilidad en el trabajo de parto, de ser posible, y elección de la postura del parto.

En España existen matronas que de forma privada atienden partos en domicilio (Alcaraz et al., 2018), siendo el coste medio unos 2500-3000 euros en función de la comunidad autónoma (Ortega Barreda et al., 2017). En los partos de bajo riesgo planificados en casa la tasa de cesáreas es cuatro veces menor que la del mismo tipo de partos en el hospital (EDUCER, 2015).

Las matronas están percibiendo un incremento de la demanda para parir en casa y, también, para el acompañamiento a domicilio durante la dilatación y las fases iniciales para reducir el tiempo de estancia en el hospital. En Cataluña, una cincuentena de mujeres ha pedido un parto en casa y se ha dicho que sí a una veintena (ACN.cat, 2020). La matrona y vocal de la Asociación Catalana de Matronas (ACL) ${ }^{1}$ y de la Asociación de Matronas de Parto en Casa de Catalunya (ALPACC) ${ }^{2}$, Alba Pallisé, ha asegurado que se ha registrado un aumento de la demanda de partos en casa y que la cifra de peticiones se ha «triplicado» durante el periodo de confinamiento por el coronavirus (NIUS, 2020).

\footnotetext{
${ }^{1}$ Asociación Catalana de Matronas (ACL): <www.llevadores.cat/>.

${ }^{2}$ Asociación de Matronas de Parto en Casa de Catalunya (ALPACC): < $\underline{\text { https://levadorespartacasa.org/en/>. }}$.
} 
El objetivo de este estudio pretende dar voz a estas mujeres y comprender cómo se revela y prospera la decisión de querer parir en casa en el contexto de la pandemia del coronavirus a partir de la vivencia de una pareja que concibe a su primer hijo.

\section{METODOLOGÍA}

Ante este contexto, se decidió llevar a cabo un estudio cualitativo (Berengera et al., 2014), con enfoque etnográfico, mediante una entrevista en profundidad realizada por una de las investigadoras de este artículo. La entrevista se desarrolló en tres encuentros de diferente duración (desde unas horas hasta un día entero) y que tuvieron lugar en la vivienda de la entrevistada a partir de la tercera semana tras el alumbramiento. La larga duración de la convivencia con la pareja permitió así mismo una observación directa, de primera mano, además de mantener conversaciones, unas veces más y otras veces menos formales, que han ido desde la charla para mantener la relación y ponerse al día de la situación, hasta las «entrevistas formales» más prolongadas.

En cada visita se estableció un clima de confianza con un diálogo espontáneo, informal al inicio e intercambio de ideas con ambos miembros de la pareja, además de realizar las grabaciones en audio de una hora y media aproximadamente en cada una, lo cual permitió obtener otra información como el lugar donde tuvo lugar el parto, el ambiente, relación y cercanía con los protagonistas en primera persona. Se usó una estrategia de investigación emic centrada en las creencias y percepciones vividas por los protagonistas (en este caso la pareja entrevistada) a la que se añadió un enfoque etic (desde la perspectiva del observador/investigador) en la interpretación de las percepciones y conclusiones derivadas con el fin de poder aplicarse a las nuevas demandas a las que deberá enfrentarse el sistema sanitario.

Se procedió a transcribir las entrevistas de forma manual por parte de la primera autora de este trabajo, ordenando de forma cronológica la información de las vivencias, decisiones y todo el recorrido desde la semana 38 de gestación hasta inmediatamente después del parto. El proceso de análisis ha consistido en ordenar, estructurar, comparar y dar significado a la información obtenida. Para aumentar la validez del procedimiento, el texto obtenido se envió a la persona entrevistada y también a la matrona que asistió el parto (coautora del estudio) para que pudieran revisar, rectificar o modular el relato final. 
De esta manera, se gestionó la triangulación de datos y también de investigadores siguiendo los criterios de calidad en metodología cualitativa (Guba y Lincoln, 2002).

Los principios éticos se guiaron por el respeto a los participantes, solicitud del consentimiento informado para las grabaciones y garantizar el anonimato tanto de la persona como de los datos obtenidos.

Presentar este estudio mediante una sola entrevista, aunque sea en profundidad, puede suponer una cierta limitación en cuanto a la extrapolación de los resultados, pero consideramos que es importante en este momento por la situación de urgencia sanitaria dar voz a la situación en que las mujeres gestantes se ven sumergidas, aunque, evidentemente, deberá complementarse con más estudios que corroboren estos resultados.

\section{Datos sociodemográficos}

Se trata de una mujer de 27 años, nacida en Cataluña, con un nivel de formación universitaria en el Grado de Fisioterapia y especial interés en el cuerpo y vida desde una perspectiva integral. Casada desde hace un año y medio con su pareja con quien convive en una población, inmersa en el parque natural de Collserola, cercana a Barcelona.

\section{RESULTADOS Y DISCUSIÓN}

En este, su primer embarazo, que ha evolucionado con total normalidad, la pareja se plantea, pese a vivir cerca de Barcelona, como mejor opción dar a luz en un Hospital comarcal, del que tienen conocimiento a través de la prensa (Europa Press, 2017), que atiende partos de forma natural respetando la intimidad en un ambiente acorde con las preferencias de ambos:

Yo desde el principio, quizás por romanticismo y porque me gustaba mucho el tema del parto natural, porque a mí me apasiona el tema del cuerpo y yo deseaba tener un parto natural, entonces busqué un hospital donde hicieran un parto natural que quiere decir un lugar que te faciliten lo básico que es darte tiempo, darte luz cálida, darte una acogida y encontré el hospital que cumplía con estos requisitos (Hospital de Martorell) que te acompañaban con aromaterapia, bajando la luz, o sea, que te favorecían el parto. Porque hay hospitales que dicen practicar el parto natural, pero tienen la luz encendida, 
el aire acondicionado en marcha, es decir, no tienen en cuenta factores que son muy importantes como la ambientación.

El hospital elegido por la pareja se ajusta a las características propias de «las casas de parto». Las casas de parto son unidades obstétricas de bajo riesgo dirigidas por matronas y que en ocasiones (como esta) se encuentran muy próximas al entorno hospitalario. Desde la década de 1990, ha habido un resurgimiento de estos centros vinculados al sistema sanitario. Los estudios señalan que dar a luz en estos centros aumenta significativamente la probabilidad de tener un parto vaginal, de no necesitar anestesia epidural y de disminuir las intervenciones médicas (Henshall, Taylor y Kenyon, 2016). No obstante, también insisten en que estas unidades deben estar dotadas de personal altamente cualificado y utilizar unos protocolos clínicos bien definidos (López y Jiménez Barragán, 2009). Las casas de parto orientan el cuidado de la mujer en un modelo holístico donde la matrona prioriza la normalidad y evita intervenciones innecesarias.

La conexión con los profesionales que la fueron atendiendo durante el embarazo fueron decisivas en muchos sentidos. La evolución del peso del bebé y la gestión de las emociones tuvo un impacto evidente y generó la confianza necesaria para las decisiones posteriores:

Sí, a mi lo que me movió es que una ginecóloga que me atendía en el embarazo, la que me hacía las ecografías que ella se ha formado en medicina integrativa, en la importancia de las emociones me aconsejó de estar muy positiva y también de que energías yo me alimentaba y a mí en el embarazo me ayudó mucho, como cuando al bebé le costaba crecer me decía «sácate los estreses» y a mi durante el embarazo todo eso me iba muy bien y cuando iba corto de peso pues yo cambiaba de chip o gestionaba estreses y decidía donde ponía la energía y después él aumentaba de peso.

Aproximadamente en la semana 38 de gestación, el escenario cambia drásticamente con el estado de alarma debido a la pandemia del coronavirus, así como la incerteza de dar a luz en las condiciones adecuadas en el lugar escogido:

Pero entonces vino el confinamiento y pensé «calla que no se complique la historia». Además, me hicieron un control y el bebé estaba bastante alto aún y en ese momento yo no me sentía segura de ir a un entorno hospitalario, aunque no tenía información de la parte sanitaria, pensé que un ambiente con todas estas circunstancias ya no era aquel que me imaginaba tranquilo que no 
favorecería el parto, de alguna manera. Por la parte de contagio, pero también por cosas que yo estaba empezando a escuchar, por ejemplo, que en San Juan de Dios estaban atendiendo unos 30 partos al día o más con 4 o 5 comadronas, «ijesto las hace ir de bólido!!». Y con tanta presión no podrían atender un parto natural como yo deseaba, otra cosa son los partos asistidos. Todo esto hablándolo con mi pareja estuvimos de acuerdo en que no podríamos ir al Hospital en medio de estas circunstancias.

Y en esas circunstancias fue cuando decidió que quería intentar dar a luz en su casa, por lo que contactó con una Asociación de Matronas para concertar una visita y ver si podía obtener alguna respuesta favorable:

En ese momento ya empecé a tramar que lo quería tener en casa y fue en la semana 39, el lunes, que conecté con las comadronas enviando un mail a la Asociación de Comadronas del Parto en Casa y me contesto una comadrona. El miércoles (me hice la ecografía) vinieron a hacer la primera visita a casa para conocernos, para ver como trabajaban y también para saber si había «feeling» con calma ya que quedaban muy pocas semanas y no me quería forzar a tomar una decisión. Pero en la visita del miércoles, ya vi que la manera de ser, de hacer, la manera de argumentar todo el procedimiento con evidencias, incluso los papeles del registro te los facilitaban, lo tenían controlado. Bueno, una gente que trabajan mucho en este tema, con mucha experiencia, están a la última de estudios de todo lo que se hablaba ellas lo corroboraban. Y por supuesto la parte del «feeling» fue muy buena, tenía que ser una persona con la que yo me pudiese sentir cómoda. O sea, en todo el rato que estuvimos aquí hablando yo me sentí muy cómoda, además como mi pareja es extranjera y la comadrona también, es francesa, y ese idioma era el que se podían comunicar a mí eso me dio mucha tranquilidad, ver que se podrían entender bien. Total, que a mí y a mi pareja nos pareció muy bien.

Las comadronas advierten que no todas las demandas se podrán atender, ya que estos partos tienen que ser de bajo riesgo y porque es «muy importante el convencimiento de la pareja; la madre lo tiene que querer y el padre le tiene que dar apoyo» (ACN.cat, 2020). En el contexto del Estado español, son ciertamente prevalentes los discursos de la evidencia científica, centrados en la seguridad frente al riesgo que supone un parto, aunque a veces se muestren resultados contradictorios y a menudo fundamentados en estudios que en realidad no llegan a conclusiones tajantes (Ortega Barreda et al., 2017). 
Además, en esa primera visita también aclararon todas las dudas y preguntas que la familia planteaba acerca del parto en casa, que manifestaron abiertamente:

Y también el poder hablar con mi familia que veían el tema de parir en casa como algo antiguo, incluso me preguntaban si estas personas tenían estudios o eran una mafia que me estaban timando, porque lo veían como algo clandestino [...], y esto es ignorancia porque actualmente esto no es así, además de comprobar como estaban al corriente de la actualidad, de los últimos estudios y que no era nada clandestino les tranquilizó además de poderles hacer sus preguntas como si se necesita un lugar estéril, todo el tema de si fuese necesario ir al Hospital y esta gente estaban preparadas con un coche y tenían contactos con diferentes hospitales. Total, que ellas resolvieron sus dudas y nosotros tuvimos claro que queríamos escoger esta opción.

La preocupación de la familia en relación con el parto en casa es evidente, ya que las dudas que plantean surgen del desconocimiento a cerca de este tipo de prácticas, verbalizando el miedo hacia los riesgos que pueden surgir en el proceso y que no son baladíes:

Con todo ello, así como el lunes en el control me dijeron que el bebé estaba muy alto y el parto podía tardar, pero el miércoles después de la visita de las comadronas el bebé empezó a bajar, quizá yo lo vivo de alguna manera romántica, pero de alguna manera el sentir confianza con ellas me relajó y el proceso del parto evolucionó y el bebé empezó a bajar. De hecho, me dijeron ese día que al bebé no le faltaba mucho. Yo a partir de ese miércoles sentí que como había una opción segura pues que el niño bajaba y ya entendí que el proceso del parto se iniciaba. A partir del sábado como me habían dicho que si sientes como cuando te viene la regla, o que necesitas la oscuridad o que no te apetece seguir las bromas y no tienes ganas de hablar. Justo fue el sábado siguiente que fuimos a ver la puesta de sol aquí cerca que sentí que quería estar en la habitación a oscuras y de hecho esa noche empezaron las contracciones que se alargaban durante el día pero que se prolongaron toda la semana. Yo ese sábado por la noche llamé a la comadrona y me dijo que intentase dormir porque o bien te puedes poner de parto esta noche o durarte toda la semana. Y efectivamente durante esa semana las contracciones venían por la noche e iban apareciendo durante la mañana. Hasta que el sábado siguiente aparecían contracciones más fuertes y esa noche las viví como dolorosas, además había leído que yo tenía que ayudar al parto y aunque las comadronas me dijeron que intentase dormir yo aquella noche no pegué ojo 
porque las contracciones eran cada vez más fuertes. Y la instrucción de que «llama a las comadronas cuando las contracciones duren un minuto y yo estaba en la cama y, claro, yo no sabía si me duraban un minuto, diez o dos. Pero yo el parto lo vivía como que tenía que ayudar, o sea, el cuerpo, que tenía que contactar con mi hijo y le preguntaba ¿si quieres salir dímelo? y notaba que el útero me apretaba.

A partir de la primera visita de las matronas, el proceso de parto se puso en marcha una semana después. No es lo habitual en los protocolos de las matronas aceptar la atención a un parto en casa en una gestación tan avanzada, ya que el contacto con la gestante se suele iniciar desde el inicio del embarazo con visitas mensuales. En este caso, debido a la situación excepcional ocasionada por la pandemia del coronavirus, todo ocurrió a partir de la 39 semana de gestación, es decir, casi al inicio del parto.

Al día siguiente conecté con la comadrona y le dije que había pasado muy mala noche y que no me veía capaz de pasar otra noche así porque no tanto por el dolor sino porque estaba bloqueada de si el bebé quería salir y lo de descansar no lo entendía, más bien tenía que ser proactiva y no lo entendía. Por la mañana sentía que todo se estaba preparando pero que yo no me acababa de poner de parto y esto me hizo remover ciertas emociones [...]. Algo que me gustó mucho de la comadrona es que me preguntó si yo tenía alguna situación o conflicto que me pudiese estar bloqueando, ¿No? Y al decirme esto pues me removió cosas que tenía que tratar o expresar o hacer salir que ahora mismo no me acuerdo de que eran pero que si me ayudo a conectarme con ciertas cosas que me estaban bloqueando un poco. Entonces me dijo, puede que esta noche tenga que venir o que todo se calme. Y esa noche todo se calmó, pero justo después durante el día siguiente ya vinieron contracciones de las que ya no puedes ni hablar y entonces fue cuando hablo con mi pareja y le dijo que yo no me tenía que preocupar y que fuese él que la llamase cuando considere que yo ya estaba de parto. Es que, claro, me dijeron que cuando yo quisiera que estuviesen a mi lado las llamase, pero por otro lado yo sabía que no hacía falta tenerlas a mi lado al inicio del parto. De todas formas, la comadrona me vino a ver y comprobó que esas contracciones ya eran de parto porque yo ya empezaba a dilatar, pero dijo, bueno, igual puede ser esta noche o la próxima. Por la tarde las contracciones seguían y la llamamos y ella me vino a ver y me trajo todo el material: la piscina, la pelota, la sillita. De nuevo dijo que había que esperar a ver si continuaba o todo se para, ese domingo por la tarde yo estaba en la fase de dilatación. 
Para las matronas, el parto en casa se fundamenta, sobre todo, en «una cuestión de confianza». Confiar en una misma, confiar en el proceso, en el cuerpo, en el bebé, confiar en quien está acompañando, y esperar que quien acompañe también se muestre seguro y confiado. En el momento del parto, la capacidad de «entrega», de «dejarse ir», de «atravesar el dolor» o de «desconectar» de pensamientos racionales se suele relacionar con la confianza en todos los elementos que participan en un parto. Esta confianza, sin embargo, no es automática, ni tampoco innata, sino que se va construyendo. Por un lado, el conocimiento previo entre profesionales y parejas sirve para generar una relación de confianza que se considera fundamental para un buen desarrollo de todo el proceso. La consideración y el respeto mutuo, ganados en los encuentros en el embarazo, permiten, como mínimo, que las parejas crean en el saber hacer profesional y las matronas en la convicción materna y paterna de tener el parto en casa. A ello se añade a menudo el establecimiento de una relación afectiva (Triuzzi, 2017).

Entonces usé la pelota que todo y estar en fase de dilatación no lo pasé tan mal y una vez aumentaban los cms. de dilatación continuaba con la piscina que esto yo no lo sé a ciencia cierta en qué momento de dilatación hay que sumergirse pero que las comadronas si lo saben. A la piscina entré dos veces, la primera que entré pues estaba allí bien, ¿pero era como que la comadrona me iba proponiendo cosas no? Como puedes ponerte así o asa o podemos ir a la piscina, es que en general a mí me ayudó mucho la comadrona además del «feeling» que teníamos pues la manera de plantear cada opción. Y sobre todo me fue muy bien que cuando estaba bloqueada fuese tan directa, como ¿hay algo que te bloquea?, o sea, no me intentase consolar, sino que de alguna manera me pusiese las cosas claras y por otra, que me diese la comodidad de que me sentía como en casa. Entonces estuve un rato en la piscina y ella me propuso ¿quieres que salgamos a dar un paseo? O si quería subir y bajar escaleras o lo que fuera, no sé, a ratos. Y entonces en la piscina, cuando me venía una contracción aquello que te comentaba de que sacaba aire o la expulsaba, me dio la sensación de que yo tenía que «empujar» como ayudar a salir y entonces me invadió una sensación de que «empujar» a mí me daba respeto, o sea, ¿el momento de que el bebé pase da respeto y fue cuando lo compartí no? Tenía miedo.

La matrona llegó a las 20h y todo eso ocurría sobre las 23h de la noche, que fue la hora que ella consideró que realmente me había puesto de parto. 
Y en ese momento las contracciones eran realmente más intensas y no era por el dolor, que iba respirando y exhalando, sino porque estaba como bloqueada, no sabía cómo ponerme y tenía la sensación como de estar reteniendo la contracción. Es decir, no tenía miedo pero había algo en mi cabeza que me bloqueaba y me dijo pues, vale salimos a dar un paseo y ella me comentó: «piensa que el espacio para que él paso ya existe, no es que la pelvis se tenga que abrir $20 \mathrm{~cm}$. y que a lo mejor si se abre es 1 o $2 \mathrm{~cm}$. máximo, o sea, piensa que el canal ya está que lo que se ensancha puede ser el final de la vagina como cuando hay desgarros pero que el suelo pélvico se ha de relajar y la tendencia es que lo tenséis cuando ha de ser al revés. Y durante el paseo también me preguntó ¿hay algo que te bloquee? Y en ese momento lo que yo sentía lo expresé y entre una cosa y la otra, al llegar me propuso ¿quieres volver a la piscina? Y le dije que sí y mientras me introducía iba pensando en no tener miedo, en no bloquearme porque antes había sentido dolor y me fui concienciando de lo que me había dicho sobre el canal que ya estaba, de alguna manera lo visualizaba todo el proceso además de que había liberado al expresar mis sentimientos. $\mathrm{Y}$ entonces, en ese momento, me pararon las contracciones y entonces me quedé con la duda, me vino otro tipo de miedo y le pregunté ¿pero si se para el parto tendré que empezar otra vez mañana? Y me dijo, no, no, todo lo que ya has hecho no se perderá, pero déjame hacerte un tacto a ver si has perdido cm. o no. Entonces me miró y me dijo, no sé qué has hecho, pero estás dilatada al completo, parirás esta noche. Al entrar en la piscina se te han parado las contracciones, mejor salir y empezar a activar y así volví a la pelota, fui a caminar por las escaleras, varias cosas y se fue reactivando todo. Además, en ese último tacto me dijo que la bolsa de líquido amniótico se había abierto y que eso ayudaría aún más al proceso de parto y que la cabeza estaba bien situada pero que no se bien que hizo, pero acabó de encararlo mejor.

En los encuentros dedicados a hablar del parto, las matronas acompañan las explicaciones de todo el proceso con ejemplos múltiples de su experiencia con otras mujeres. Se pretende transmitir, entre otras cosas, que cada parto es diferente, que puede no doler pero que en la mayoría de los casos duele, que el dolor constituye solo una mínima parte de una vivencia mucho más compleja, que la perfección no existe y que cada una lo vivirá a su manera, pudiendo siempre constituir una fuente de aprendizaje.

A continuación, me dijo, vale, ahora las contracciones no te harán tanto daño porque son las de expulsión que son diferentes a las de dilatación que son muy agudas, pero son diferentes. Yo en la expulsión pasé dolor intenso igual, 
pero lo viví diferente, no sé, en el momento en que el bebé se encaja te duele todo el sacro o también el probar diferentes posiciones, me acuerdo de que al sentarme en el taburete sentía mucho dolor en el sacro por la presión de la criatura. Y una cosa que me ayudo es cuando le pregunte ¿pero en el expulsivo las contracciones serán más fuertes? Y me contestó, serán más intensas, pero a la vez tendrás más fuerza para responder y esto me dio coraje, me ayudó a conectar. Fui probando diferentes posiciones, ahora con la pelota, luego estirada, también con el taburete y tener a alguien delante para hacer presión ya fuese mi pareja o la comadrona para empujar mejor cuando venía la contracción y al final, ¡empujando estuve 3 horas! Claro, el diámetro de la cabeza del bebé era grande, como vimos después y esto es un factor que dificulta que salga el bebé. Pues eso, fue ir probando diferentes posiciones y yo que estaba como reventada y al final, la mejor posición fue la de estirada en la cama, en decúbito lateral, con la pierna haciendo presión contra la comadrona. Lo más importante fue la tranquilidad de saber que estás con gente muy profesional, de que hay un «feeling» mutuo, las respuestas ante cada situación de bloqueo que tuve y como las gestionaron. Ellas ya detectan la situación del parto y si hubiesen visto cualquier riesgo habrían actuado.

\section{CONCLUSIONES}

La idea fundamental que sustenta la decisión de un parto en casa es la convicción de que las mujeres pueden y saben parir, y que los bebés pueden y saben nacer. Del parto se habla en términos de poder, de fuerza, de misterio, de oportunidad de crecimiento y de aprendizaje, de un repaso repentino de todo lo vivido o de un «viaje» por la existencia vivida. La experiencia de parir y de acompañar un parto se interpreta como una demostración de las capacidades humanas, de la energía y de las potencialidades de cada persona. Parir se convierte, sobre todo para ellas, en una constatación de su propia fuerza. Después del parto no es raro que las mujeres se sientan reforzadas en su autoestima, más capaces de enfrentarse a situaciones difíciles, más «poderosas».

Tras esta crisis sanitaria y la situación de miedo y ansiedad a la que las mujeres gestantes se han visto sometidas, además del riesgo biológico que puede suponer que una mujer sana y su pareja deban convivir en un medio hospitalario en el que se atienden a enfermos infecciosos, las autoridades sanitarias deberían replantearse si los hospitales son el mejor escenario para un parto que se considere de bajo riesgo, ya que la pandemia del coronavirus ha puesto de relieve y en el punto de mira otras deficiencias detectadas 
anteriormente derivadas de la alta tecnología y medicalización de los partos en general. Cada vez son más las voces que reclaman una humanización de la asistencia, en general, y de los procesos de parto en entornos más naturales, asistidos de forma segura, pero sin olvidar el protagonismo y la capacidad de decisión de las propias mujeres en todo el proceso. Una tarea más a la que tendremos que enfrentarnos en un futuro, esperemos, no muy lejano. 


\section{BIBLIOGRAFÍA}

Acn.CAT. Parir en casa, la solución contra el coronavirus. En: Diari Més Digital [en línea]. 29 marzo de 2020. [Consulta: 22 mayo 2020]. Disponible en: <www.diarimes.com/es/noticias/actualidad/cataluna/2020/03/29/parir_casa_solucio n_contra_coronavirus_79265_3029.html? >.

AlCARAZ, L., et al. Guía de asistencia al parto en casa [en línea]. Barcelona: Associació Catalana de Llevadores, 2018. [Consulta: 22 mayo 2020]. Disponible en:

<llevadorespartacasa.org/wp-content/uploads/2018/06/Guia_PartoCasa_2018.pdf>.

ArAúJo, B. R. O., et al. Entre ritos e contextos: Decisões e significados atribuídos ao parto natural humanizado. En: Cultura de los Cuidados. 2019, vol. 23, núm. 54, pp. 206-216.

BerenguerA, A. Escuchar, observar y comprender. Recuperando la narrativa en las Ciencias de la Salud. [en línea] Barcelona: Generalitat de Catalunya, Departament de Salud, 2014. Disponible en:

<saludcomunitaria.files.wordpress.com/2014/12/escucharobservarcomprender.pdf>.

BIURRUn GARRIDO, A. La humanización de la asistencia al parto: Valoración de la satisfacción, autonomía y del autocontrol [en línea]. [Tesis doctoral]. Barcelona: Universidad de Barcelona, 2017. [Consulta: 3 julio 2020]. Disponible en: $<$ https://www.tesisenred.net/bitstream/handle/10803/457137/ABG_TESIS.pdf?sequ ence $=1 \&$ is Allowed $=\mathrm{y}>$.

CORES, N. F. Nacer en tiempos de coronavirus: «Pedí quitarme la mascarilla porque no podía respirar. Les dije: "Yo así no puedo parir”». En: 20 Minutos. Salud [en línea]. 14 abril 2020. [Consulta: 22 mayo 2020]. Disponible en: $<$ https://www.20minutos.es/noticia/4225644/0/nacer-en-tiempos-de-coronavirus/>.

CulP, W. C. Coronavirus Disease 2019. En: A \& A Practice. 2020, vol. 14, núm. 6.

EDUCER. El parto en casa planificado. En: El blog del parto en casa [en línea]. 2015. [Consulta: 2 junio 2020]. Disponible en:

$<$ https://www.elpartoesnuestro.es/informacion/parto/el-parto-en-casa-planificado>. EFE, 2020. Parir en tiempos de coronavirus: dos madres cuentan su experiencia. En: $A B C$ [en línea]. 31 marzo 2020. [Consulta: 22 mayo 2020]. Disponible en: $<$ www.abc.es/familia/bebes/abci-parir-tiempos-coronavirus-madres-cuentanexperiencia-202003310150_noticia.html>. 
EuROPA PRESS, 2017. Un hospital de Martorell abrirá una casa de partos gestionada sólo por matronas. En: La Sexta.com [en línea]. 2017. [Consulta: 8 julio 2020]. Disponible en: $\quad<$ www.lasexta.com/noticias/sociedad/hospital-martorell-abrira-casa-partosgestionada-solo-matronas_201705265927f4770cf20292e7dffd5c.html>.

Federación De Asociaciones De Matronas De España. La evidencia científica lo avala: el parto en casa es seguro [en línea]. Madrid: FAME, 2017. Disponible en: <apromap.com/nota-de-prensa-fame-parto-\%0Aen-casa/>>

Federación De Asociaciones De Matronas De España. Iniciativa Parto Normal [en línea]. [Consulta: 29 mayo 2020]. Madrid: FAME, 2011. Disponible en: $<$ www.federacion-matronas.org/informacion-de-interes-paraprofesionales/iniciativa-parto-normal-de-la-fame/>.

FERNÁNDEZ, F. Coronavirus y parto, las mujeres mantienen su derecho a decidir. Primeros protocolos de partos en la Comunidad de Madrid al principio del COVID19 [en línea]. En: Francisca Fernández Guillén [Blog]. 2020. Disponible en:

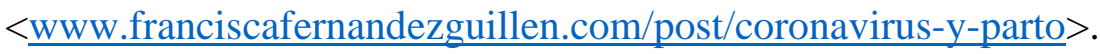

FrANCO, L. El miedo al contagio por coronavirus aumenta el interés por los partos en casa. En: El País [en línea]. 21 mayo 2020. [Consulta: 22 mayo 2020]. Disponible en: <elpais.com/elpais/2020/05/21/mamas_papas/1590040313_226582.html>.

GOBERnA TRICAS, J. Dones i procreació: ètica de les pràctiques sanitaries i la relació asistencial en embaràs i naixement [en línea]. [Tesis doctoral]. Barcelona: Universitat de Barcelona, 2009. [Consulta: 3 julio 2020]. Disponible en: <https://www.tesisenred.net/handle/10803/1762\#page=1>.

GuBA, E.; LiNCOLN, Y. Paradigmas en competencia en la investigación cualitativa. En: Denman, C.; Haro, J. A. (eds.). Por los rincones. Antología de métodos cualitativos en la investigación social. Hermosillo, Sonora: El Colegio de Sonora, 2002, pp. 113145.

Henshall, C.; TAYlor, B.; Kenyon, S. A systematic review to examine the evidence regarding discussions by midwives, with women, around their options for where to give birth. En: BMC Pregnancy and Childbirth. 2016, vol. 16, núm. 1.

KoninKLiJKe Nederlandse ORGANisatie VAN VerLOSKundigen (KNOV). Midwifery in the Netherlands [en línea]. 2020. [Consulta: 22 mayo 2020]. Disponible en: $<$ http://www.knov.nl/samenwerken/tekstpagina/489-2/midwifery-in-thenetherlands/hoofdstuk/463/midwifery-in-the-netherlands/>. 
LÓPEZ, G.; JimÉNEZ BARRAGÁN, M.. Las casas de parto dentro del sistema hospitalario. En: Matronas profesión. 2009, vol. 2, pp. 12-15.

Martínez-Mollá, T. M.; SiLes, J.; Solano, M. Evitar la violencia obstétrica: motivo para decidir el parto en casa. En: MUSAS. 2019, vol. 4, núm. 2, pp. 53-77. Disponible en: <https://doi.org/10.1344/musas2019.vol4.num2.4>.

NiUS. Se triplica la demanda de partos en casa durante el confinamiento en Cataluña. En: Niusdiario.es [en línea]. 9 mayo 2020. [Consulta: 29 mayo 2020]. Disponible en: $<\underline{\text { https://www.niusdiario.es/sociedad/sanidad/triplica-demanda-partos-casa- }}$ confinamiento-cataluna_18_2943945026.html>.

O’CONNELL, M., et al. Midwives in a pandemic: A call for solidarity and compassion. En: Women and Birth. 2020, vol. 33, núm. 3, pp. 205-206.

OlzA, I. Sanar el trauma del parto en tiempos de pandemia. En: iboneolza.org [en línea]. 14 mayo 2020. [Consulta: 22 mayo 2020]. Disponible en: <iboneolza.org/2020/05/14/sanar-el-trauma-del-parto-en-tiempos-de-pandemia/> .

Organización Mundial De La SALUd (OMS). Prevencion y erradicacion de la falta de respeto y maltrato en el parto. [en línea]. Ginebra: OMS, 2014. Disponible en: $<$ www.who.int/reproductivehealth/topics/maternal_perinatal/statementchildbirth/es/>.

ORGANIZACión Mundial DE LA SALUd (OMS). Statement on caesarean section rates [en línea]. Ginebra: OMS, 2015. Disponible en:

$<$ www.who.int/reproductivehealth/publications/maternal_perinatal_health/csstatement/en/>.

ORTEGA BARREDA, E., et al. Panorámica internacional en relación a las recomendaciones, práctica clínica y legislación del parto en casa. En: ENE Revista de Enfermería. 2017, vol. 11, núm. 1.

PinTo, I. Parto domiciliario, ¿una elección de futuro? [Trabajo Fin de Grado]. Jaén: Universidad de Jaén, 2016. Disponible en: http://tauja.ujaen.es/bitstream/10953.1/2882/1/Inmaculada_Pinto_Ortiz_TFG.pdf

Ramírez, C. Parto en Casa Planificado con Asistencia Profesional: Recuperando el Protagonismo. [Memoria Fin de Grado] Santiago de Chile: Universidad de Chile, 2015. Disponible en:

<http://repositorio.uchile.cl/bitstream/handle/2250/140306/tesis\%20carla\%20ramire z\%20\%281\%29\%20\%281\%29.pdf?sequence=1\&isAllowed=y > . 
Ruiz BERDún, D. Parir en tiempos de coronavirus: por qué en el Sistema de Salud español solo se puede dar a luz en hospitales. En: The Conversation [en línea]. 23 abril 2020. [Consulta: 31 mayo 2020]. Disponible en: <https://theconversation.com/parir-entiempos-de-coronavirus-por-que-en-el-sistema-de-salud-espanol-solo-se-puede-dara-luz-en-hospitales-135711>.

TRIUZzI, S. Parir en casa. Un estudio antropológico de la atención domiciliaria al parto en el contexto andaluz. [en línea]. Sevilla: Universidad de Sevilla, 2017. [Consulta: 28 mayo 2020]. Disponible en:

$<$ https://idus.us.es/xmlui/handle/11441/69709>.

VIVAs, E., 2020. El parto en tiempos de covid-19. En: El Periódico [en línea]. 15 abril 2020. [Consulta: 22 mayo 2020]. Disponible en:

<www.elperiodico.com/es/opinion/20200415/embarazo-parto-coronavirus-covid19-esther-vivas-7928942>. 